\title{
Hydrothermal Assisted Synthesis of Hierarchical Nanostructured Metal Oxide Thin Film
}

Bhosale PN ${ }^{1 *}$, Kondalkar VV1, Mane RM ${ }^{1}$, Choudhury Sipra² and Khot KV ${ }^{1}$

${ }^{1}$ Department of Chemistry, Materials Research Laboratory, Shivaji University, Kolhapur, India ${ }^{2}$ Chemistry Division, Bhabha Atomic Research Centre (BARC), Mumbai, India

\section{Introduction}

Hydrothermal synthesis has been most popular one, gathering interest from scientist and technology, particularly in the last fifteen years. Hydrothermal technology not only helps in synthesis of mono dispersed but also highly homogeneous nanoparticles. The term hydrothermal is purely geological and it was first used by the British geologist, Sir Roderick Murchison to describe the action of water at levitated temperature and pressure [1]. Hydrothermal processing can be defined as many heterogeneous reaction in the presence of aqueous solvent or mineralizes and re-crystallize material that are relatively insoluble conditions is usually performed below supercritical temperature of water $\left(374^{\circ} \mathrm{C}\right)$ [2]. The hydrothermal methods can be used to prepare much geometry including thin film, single crystal, nanocrystals, and bulk powder. In addition morphology sphere (3D), nanosheets (2D) and wire, rods (1D) of the crystal is controlled by manipulating the solvent [3-5], chemical of interest concentration and kinetics control. The technique can be used to synthesize thermodynamically stable and metastable states including novel materials that cannot be easily formed by other synthetic route. The hydrothermal synthesis is carried out in autoclaves which is sealed steel cylinder that can withstand at high temperature and pressure for a long time [6-8]. In hydrothermal synthesis materials takes for a longer reaction time. The autoclave must be high temperature and pressure so the use most successful 316 series stainless steel, iron, nickel, cobalt based super alloy is used. To avoid corrosion of autoclave materials is coated with non-reactive Teflon materials.

\section{Experimental setup for hydrothermal synthesis}

The hydrothermal reactions are carried out at elevated pressure so specialized equipment is essential. Under such condition it is easy to work with sealable vessels. The vessel is referred as autoclaves or hydrothermal bomb that withstands in harsh chemical conditions. Due to the high pressure, such devices are usually manufactured from metal alloys. Stainless steel bombs are most common, but other materials like tantalum, hastelloy or titanium are employed as well for more corrosive liquids. The autoclaves had an inner wall coated with Teflon cylinder that is chemically inert. Hydrothermal system is shown in Figure 1. The autoclave was filled $80 \%$ to its total volume and sealed. The autoclave was heated, resulting in increase in the autogeneous pressure of solvent.

External pressure adjustment is not possible for we used system. The critical point for water lies at $374^{\circ} \mathrm{C}$ and 218 atm. Water is become supercritical above this temperature and pressure. Supercritical fluids show characteristics of both a liquid phase and a gas phase. There is very less surface tension at the interfaces of solids and supercritical fluids, so supercritical fluids exhibit high viscosities and easily dissolve chemical compounds that would be exhibit very low solubility's under ambient conditions. A hydrothermal process is simple take advantage of the increased solubility and reactivity of reactants at elevated temperatures and pressures without bringing the solvent to its critical point.

\section{Factors affecting hydrothermal technique}

Hydrothermal growth of different nanostructures depends on many factors such as deposition temperature, nature and concentration of precursors, addition of surfactants, solution $\mathrm{pH}$ value, crystal properties of the materials and nature of the substrate.

\section{Solution $\mathrm{pH}$ value}

The surface charge density of metal oxide will change the interfacial energy and affect the size and distribution of the nanostructures. When solution $\mathrm{pH}$ changed results in adsorption of protons or hydroxyl groups will change the surface charge density. So change in solution $\mathrm{pH}$ result in different charge densities and electrostatic forces which affect the aggregation and ripening of the nanostructures.

\section{Concentrations of the reactants}

The growth rate and quality of the thin film deposition was greatly influences by the nature and concentration of the reacting species. The growth kinetics also depends on the nature of reactants. The concentrations of the reactants have impact on the morphology of final product of nanostructures.

\section{Addition of surfactants}

When the precursors are mixed with organic surfactants, then formation of monomers takes place. As the monomers achieve the critical concentration (nucleation threshold), aggregation occurs to form nuclei. After formation of nuclei, the monomers concentration deceases below the critical concentration, and then the monomers can only add to existing nuclei for continuous growth of thin film.

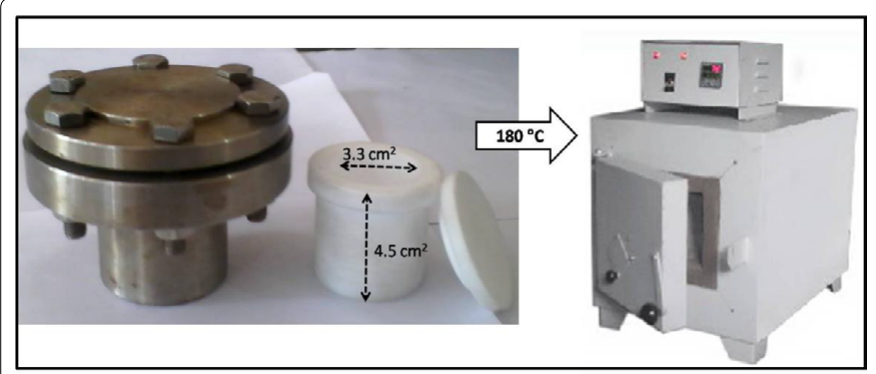

Figure 1: Photograph of hydrothermal set up used for synthesis of hierarchical nanostructured metal oxide thin film.

*Corresponding author: Bhosale PN, Department of Chemistry, Materials Research Laboratory, Shivaji University, Kolhapur-416004, India, Tel: 9420007500 ; E-mail: p_n_bhosale@rediffmail.com

Received June 23, 2015; Accepted June 27, 2015; Published July 07, 2015

Citation: Bhosale PN, Kondalkar VV, Mane RM, Choudhury Sipra, Khot KV (2015) Hydrothermal Assisted Synthesis of Hierarchical Nanostructured Metal Oxide Thin Film. J Nanomed Nanotechnol 6: e134. doi:10.4172/2157-7439.1000e134

Copyright: @ 2015 Bhosale PN, et al. This is an open-access article distributed under the terms of the Creative Commons Attribution License, which permits unrestricted use, distribution, and reproduction in any medium, provided the original author and source are credited. 
Citation: Bhosale PN, Kondalkar VV, Mane RM, Choudhury Sipra, Khot KV (2015) Hydrothermal Assisted Synthesis of Hierarchical Nanostructured Metal Oxide Thin Film. J Nanomed Nanotechnol 6: e134. doi:10.4172/2157-7439.1000e134

\section{Nature of the substrate}

In addition dimensional control of hydrothermally grown oxide nanostructures attract a lot of attention. It has been proposed that the control of the interfacial tension will affect the thermodynamics and kinetics of the nucleation and growth. The substrate nature affects the nucleation central and control the crystal growth in desired direction to the substrate to form a desired morphology for example control of nanorods perpendicular to substrate surface or parallel to the substrate surface.

\section{Deposition temperature}

To study the influence of synthesis temperature on the phase and morphology of metal oxides was prepared at different temperature while keeping other parameter constant. There is a possibility to developed well crystalline phase and decrease in the unit-cell volume. As deposition temperature increases the dissociation of complex increases. The kinetic energy of molecules also increases leading to greater interaction between ions which results into precipitation and give grained structure of thin films.

\section{Deposition time}

When the film growth was performed for different hours, noticeable difference in morphology or crystallite density was observed. This reveals that the majority of nucleation, growth of nanoparticles and large crystallites occurs within the few hours. The morphology can be tuned in desired shape e.g. nanorods with either pointed tipsor flat tops can be produced by varying the deposition time.

In some cases, it is better to use the experiential rule which agrees with the fact that solubility becomes high in solvents with higher dielectric constant and types of chemical bond which are closer to solute substance. Deviation from this group has a place in the case where specific interaction between solid substance and the solvent occurs. The synthesis and recrystallization of a compound and growth of single crystals on seed are all carried out using different solvents on the basis of physico-chemical considerations. The following conditions are used in selecting the most suitable mineralizers:

1. The formation of readily-soluble mobile complexes in the solution.

2. A fairly sharp change in the solubility of the compounds with changing temperature or pressure.
3. A specific quantitative value of the absolute solubility of the compound being crystallized.

4. A specific redox potential of the medium ensuring the existence of ions of the required valence.

5. Analogy of the dissolution of the test compounds.

\section{Conclusions}

Hydrothermal synthesis method is a promising approach for the fabrication of low dimensional hierarchical structured metal oxide thin films. The hydrothermal route is facile, has control over the size, requires low reaction temperatures, and is economic for large scale production of materials with large surface areas and unique morphology.

\section{Acknowledgement}

One of the authors Vijay V. Kondalkar wishes to acknowledge the DAE-BRNS, Mumbai, for financial support through the DAE-BRNS Major Research Project no. 2012/34/51/BRNS/2036

\section{References}

1. Sir Roderick Murchison (1840s)

2. Morey GW, Niggli P (1913) The Hydrothermal Formation of Silicates, A Review. J Am Chem Soc 35: 1086.

3. Lin K, Chang J, Zhu Y, Wu W, Cheng G, et al. (2009) A Facile One-Step Surfactant-Free and Low-Temperature Hydrothermal Method to Prepare Uniform 3D Structured Carbonated Apatite Flowers. Crystal Growth and Design 9: 177-181.

4. Patil PB, Mali SS, Kondalkar VV, Pawar NB, Khot KV, et al. (2014) Single step hydrothermal synthesis of hierarchical $\mathrm{TiO}_{2}$ microflowers with radially assembled nanorods for enhanced photovoltaic performance. RSC advances 4: 47278-47286

5. Patil PB, Mali SS, Kondalkar VV, Khot KV, Mane RM, et al. Journal of Materials Science: Materials in Electronics.

6. Yang Q, Lu Z, Liun J, Lei X, Chang Z, et al. (2013) Metal oxide and hydroxide nanoarrays: Hydrothermal synthesis and applications as super capacitors and nanocatalysts. Prog Nat Sci: Mater Inter 23: 351-366.

7. Kondalkar VV, Mali SS, Kharade RR, Khot KV, Patil PB, et al. (2015) High performing smart electrochromic device based on honeycomb nanostructured h-WO3 thin films: hydrothermal assisted synthesis. Dalton Trans 44: 2788-2800.

8. Kondalkar VV, Kharade RR, Mali SS, Mane RM, Patil PB, et al. (2014 Nanobrick-like $\mathrm{WO}_{3}$ thin films: Hydrothermal synthesis and electrochromic application. Superlattices and Microstructures 73: 290-295. 